\title{
CHAOTIC BREAKDOWN OF A PERIODICALLY FORCED, WEAKLY DAMPED PENDULUM
}

\author{
PETER J. BRYANT ${ }^{1}$
}

(Received November 2, 1990; revised 21 March 1991)

\begin{abstract}
An investigation is made of the transition from periodic solutions through nearlyperiodic solutions to chaotic solutions of the differential equation governing forced coplanar motion of a weakly damped pendulum. The pendulum is driven by horizontal, periodic forcing of the pivot with maximum acceleration $\epsilon g$ and dimensionless frequency $\omega$. As the forcing frequency $\omega$ is decreased gradually at a sufficiently large forcing amplitude $\epsilon$, it has been shown previously that the pendulum progresses from symmetric oscillations of period $T(=2 \pi / \omega)$ into a symmetrybreaking, period-doubling sequence of stable, periodic oscillations. There are two related forms of asymmetric, stable oscillations in the sequence, dependent on the initial conditions. When the frequency is decreased immediately beyond the sequence, the oscillations become unstable but remain in the neighbourhood in $(\theta, \dot{\theta})$ phase space of one or other of the two forms of periodic oscillations, where $\theta(t)$ is the pendulum angle with the downward vertical. As the frequency is decreased further, the oscillations move intermittently between the neighbourhoods in $(\theta, \dot{\theta})$ phase space of each of the two forms of periodic oscillations, in paired nearly-periodic oscillations. Further decrease of the forcing frequency leads to time intervals in which the motion is strongly unstable, with the pendulum passing intermittently over the pivot, interspersed with time intervals when the motion is nearly-periodic and only weakly unstable. The strongly-unstable intervals dominate in fully chaotic oscillations. Windows of independent, stable, periodic oscillations occur throughout the frequency range investigated. It is shown in an appendix how the Floquet method may be interpreted to describe the linear stability of the periodic and nearly-periodic solutions, and the windows of periodic oscillations in the investigated frequency range are listed in a second appendix.
\end{abstract}

\section{Introduction}

A pendulum forced by a horizontal acceleration $\epsilon g \sin \omega t$ of its pivot has

\footnotetext{
'Department of Mathematics, University of Canterbury, Christchurch, New Zealand. (C) Copyright Australian Mathematical Society 1992, Serial-fee code 0334-2700/92
} 
the equation of motion

$$
\ddot{\theta}+2 \delta \dot{\theta}+\sin \theta=\epsilon \cos \theta \sin \omega t,
$$

where $\theta$ is the angular displacement from the downward vertical, $\delta$ is the damping ratio (actual/critical), $\omega$ is the ratio of the forcing frequency to the natural frequency, and the unit of time is the inverse natural frequency. The periodic solutions of (1.1) have been investigated previously [3], where examples are presented of symmetry-breaking, period-doubling sequences of solutions. One such sequence, at constant $\epsilon=1.2$ as $\omega$ is changed, is described in terms of resonance curves and Poincarè cross-sections in Figures 5 and 6 of [3] respectively. This sequence is continued here beyond the periodic motion into chaotic breakdown. Following [3], the damping ratio is $\delta=1 / 8$ in the numerical calculations. Resonance curves are defined as plots of $\langle E\rangle^{1 / 2}$ versus $\omega$, where

$$
E=\dot{\theta}^{2} / 2+1-\cos \theta
$$

is a measure of the energy of oscillations, and \langle\rangle denotes a mean over a complete period of periodic oscillations, or over a specified number of forcing periods for nearly-periodic oscillations.

The calculation of the periodic solutions is based on numerical collocation applied to truncated Fourier expansions for $\theta(t)$ [3]. The nonperiodic asymptotic solutions in time of (1.1) are calculated using the NAG subroutine D02EBF for step-by-step integration of the equivalent pair of first-order differential equations, with a local error tolerance of $10^{-11}$.

The torque-driven damped pendulum, whose equation of motion, in the above notation, is

$$
\ddot{\theta}+2 \delta \dot{\theta}+\sin \theta=\epsilon \cos \omega t,
$$

is used by Baker and Gollub [1] as the primary model for an excellent introduction to chaotic dynamics. Many of the properties described there are relevant to the horizontally forced, damped pendulum investigated here, and are compared below. The emphasis here is on a qualitative description and understanding of chaotic breakdown, rather than on the calculation of the usual averaged quantitative descriptors such as Lyapunov exponents or attractor dimensions, for which reference is made to [1].

Both problems are much simplified models for the oscillations of a damped dynamical system such as a mechanical structure, a fluid in a container, or an electrical circuit, that is subjected to periodic forcing. Parallels with the periodic structure of the wake behind a bluff body in a flowing fluid are discussed in Section 7. One reason for the present investigation into the planar oscillations of a forced pendulum is that there is then only one resonant mode 
of oscillations, but as is indicated above, a large variety of types of nonlinear oscillations is still possible even for this simple model.

\section{Periodic oscillations}

Oscillations of the pendulum with a period $m T(T=2 \pi / \omega)$ are represented by the Fourier series

$$
\theta=\sum_{k=0}^{N}\left[a_{k} \cos (k \omega t / m)+b_{k} \sin (k \omega t / m)\right]
$$

where the number of harmonics, $N$, is chosen to be sufficiently large that (2.1) satisfies (1.1) to any required precision (typically $10^{-4}$ ). The perioddoubling sequence when $\epsilon=1.2$ is illustrated in Figure 1 (see next page) (adapted from [3], Figure 5), where resonance curves are drawn for the different forms of periodic, asymmetric oscillations from period $T$ to period $16 T$. The stable sections of the resonance curves are solid and the unstable sections are dotted. The curve on the right is a portion of the resonance curve for $T$-periodic oscillations. The resonance curve for $2 T$-periodic oscillations begins at the first period-doubling point $\omega=0.8828$. It bifurcates to the resonance curve for $4 T$-periodic oscillations at $\omega=0.8732$, then to the resonance curve for $8 T$-periodic oscillations at $\omega=0.8712$. A stable $16 T$-periodic oscillation occurs at $\omega=0.8708$. The resonance curves appear to be leapfrogging towards a limiting curve as the period multiplicity increases. The $\omega$-bandwidth ratios are 4.8 and 5.0, in reasonable agreement with Feigenbaum's universal ratio 4.67 [4]. Feigenbaum's ratio is derived for one-parameter systems, but ratios here can be calculated for either $\omega$ bandwidths or $\epsilon$-bandwidths (or even for $\delta$-bandwidths). If the bandwidth ratio is applicable to the remainder of the period-doubling sequence, the sequence should terminate before $\omega=0.8707$. The asymptotic solution of (1.1) at $\omega=0.8707$ is found to remain nearly-periodic, without tending to exactly periodic oscillations.

The phase-plane diagram $\dot{\theta}$ against $\theta / \pi$, for the stable $16 T$-periodic oscillations at $\omega=0.8708$ is shown in Figure 2 (see page 157). The path closes after 16 circuits of the origin. It should be noted that there is a second solution whose diagram is the same as Figure 2 except that it is reflected about $\theta=0$, because if $\theta(t)$ is a solution of (1.1) so is $-\theta(t+T / 2)$, and the associated reflection about $\dot{\theta}=0$ leaves the figure unchanged. The asymptotic oscillations in time are determined by the initial conditions. 


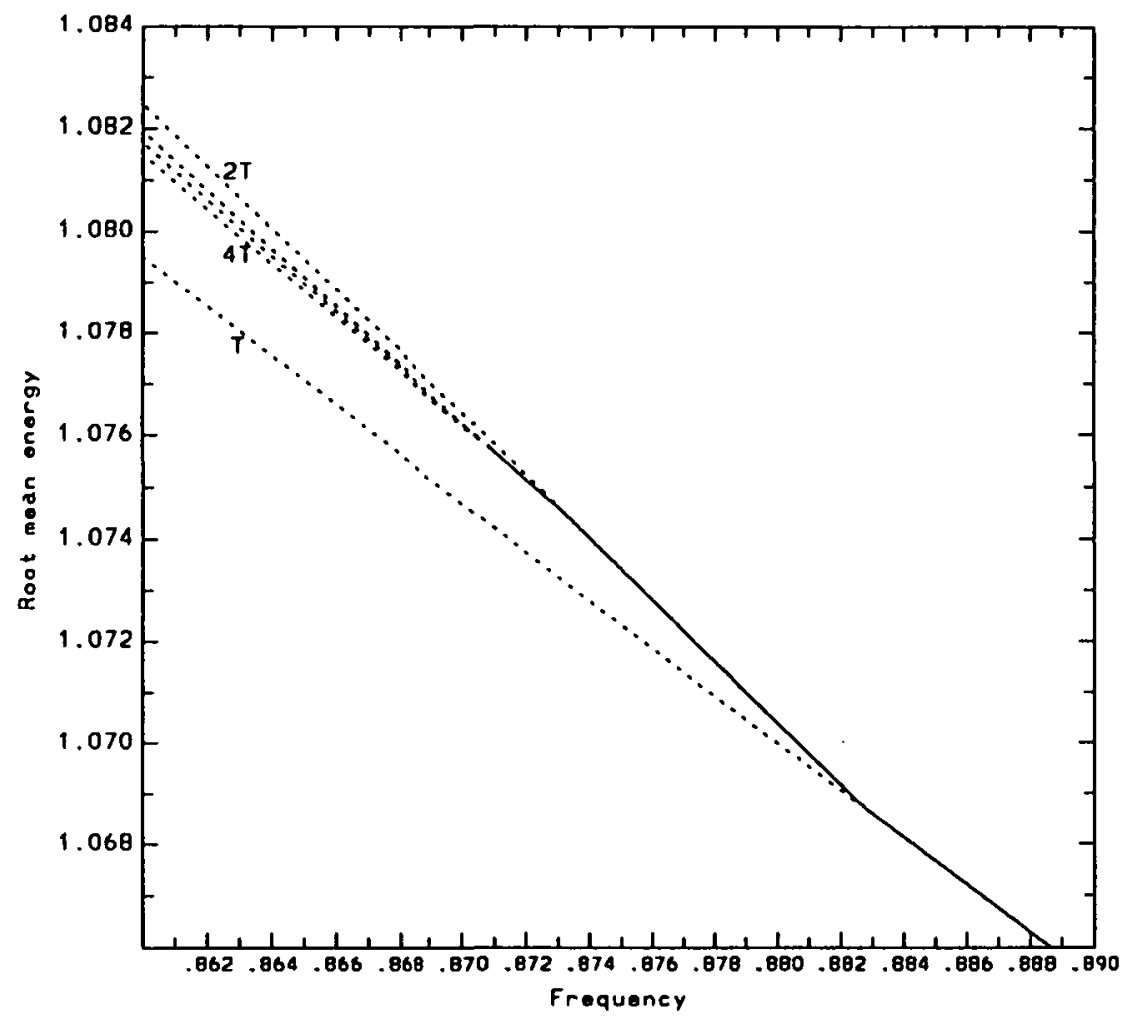

FIGURE 1. Resonance curves for the period-doubling sequence of asymmetric oscillations with periods $T, 2 T, \ldots, 16 T$. The order of the curves, from the top, is $2 T, 8 T, 16 T, 4 T$, and $T$.

Although the asymptotic solution is nearly periodic when $\omega$ is reduced from 0.8708 to 0.8707 , it reverts to being exactly periodic with a period of $20 T$ when $\omega$ is reduced again to 0.8706 . The $20 T$-periodic oscillations have a very small window of stability, with the resonance curve having a turning point between 0.8706 and 0.8707 on one side, and becoming unstable (presumably through a period-doubling sequence) between 0.8706 and 0.8705 on the other side. The phase-plane diagram for the stable $20 T$ periodic oscillations at $\omega=0.8706$ is very similar to that in Figure 2, but the two periodic solutions are believed to be independent. Other small windows of sta' $^{\prime} \cdot$ periodic oscillations are described in Appendix B. 


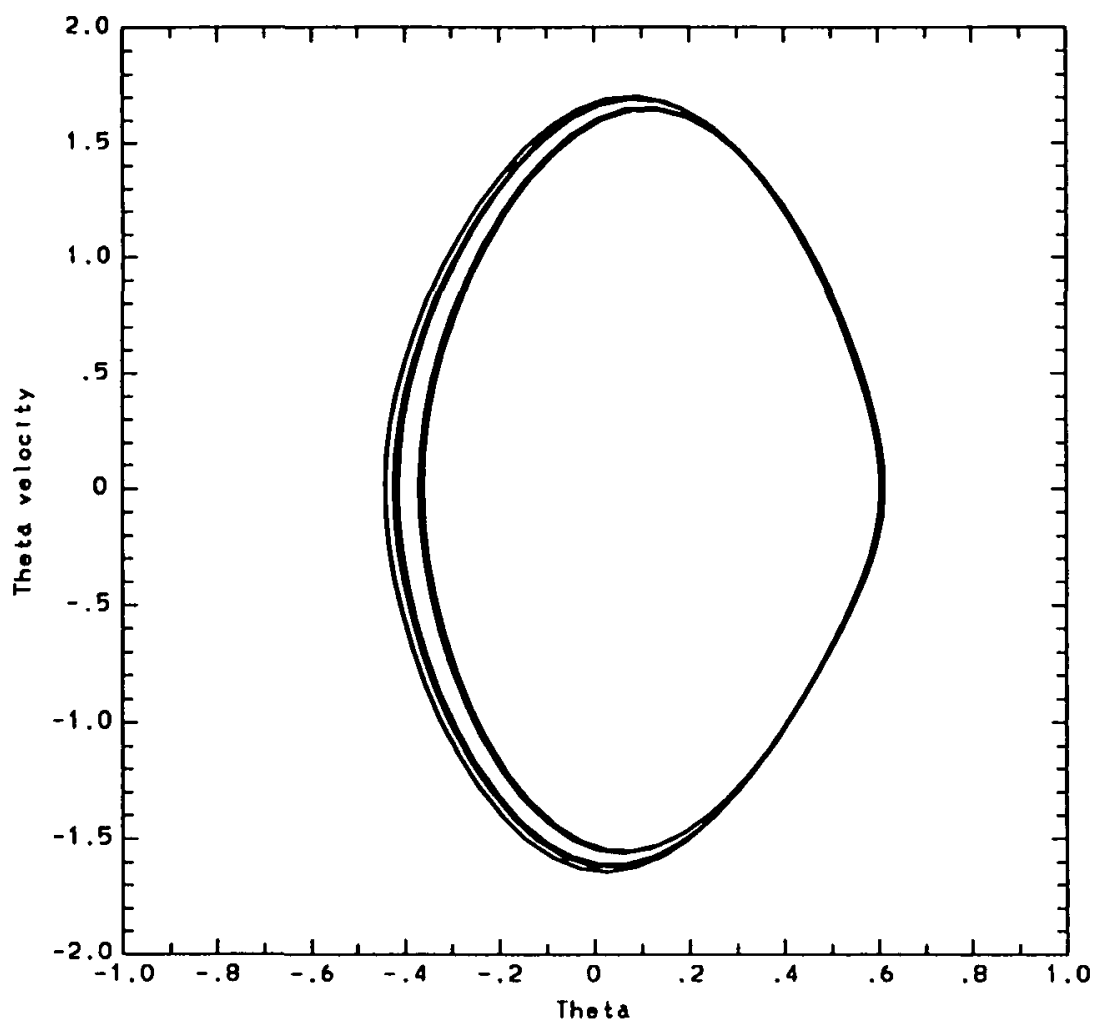

Figure 2. The phase-plane diagram, $\dot{\theta}$ against $\theta / \pi$, for the $16 T$-periodic oscillation with $\epsilon=1,2, \omega=0.8708$, and $\delta=0.125$.

\section{Nearly-periodic oscillations}

The oscillations at frequencies in a neighborhood beyond the end of the period-doubling sequence of Figure 1 are nearly-periodic, except for the small window of $20 T$-periodic oscillations described above. The phase-plane diagram, $\dot{\theta}$ against $\theta / \pi$, for 100 forcing periods of the nearly-periodic oscillations at $\omega=0.8700$ is drawn in Figure 3 (see next page). The path never closes, but always lies near the closed path of Figure 2. Also, like the periodic solution in Figure 2, there is a second set of nearly-periodic oscillations at this frequency whose diagram is similar to Figure 3 except that it is reflected about $\theta=0$.

The periodic oscillations whose resonance curves are drawn in Figure 1 have unstable solutions near $\omega=0.8700$, suggesting that one method for analysing the nearly-periodic oscillations is to compare their spectral compositions with those of the unstable periodic oscillations at the same frequency. 


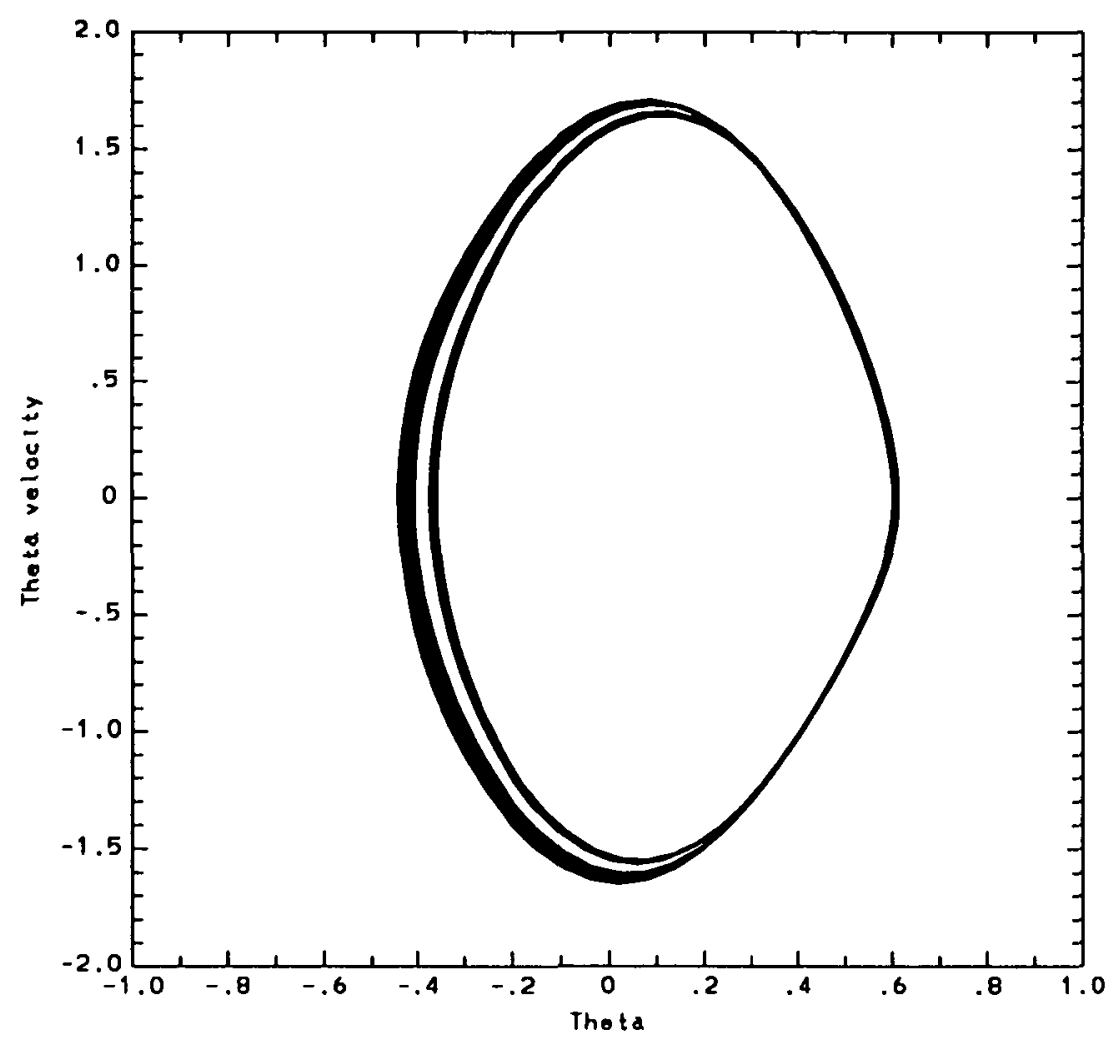

Figure 3. The phase-plane diagram, $\dot{\theta}$ against $\theta / \pi$, over 100 forcing periods with $\epsilon=1.2$, $\omega=0.8700$, and $\delta=0.125$.

Because the spectral components of the periodic oscillations are in phaselocked relationships with the periodic forcing, a suitable comparison of spectral compositions can reveal the extent of phase-locking in the nearly-periodic oscillations. Consecutive $16 T$-intervals of the nearly-periodic oscillations were calculated, and an ensemble average taken over these consecutive intervals to extract the phase-locked part of the oscillations at integer multiples of the frequency $\omega / 16$. The result is an averaged $16 T$-interval whose spectral composition described the phase-locking in the nearly-periodic oscillations at the given parameter values.

The comparison of the Fourier amplitudes at $\omega=0.8700$ is illustrated in Figure 4 . The crosses, connected by lines, include all the spectral components of the averaged $16 T$-interval whose magnitudes exceed 0.01 . Since these components are all integer multiples of $\omega / 2$, they are compared with the spectral components of the unstable $2 T$-periodic solution at $\omega=0.8700$, denoted by circles in Figure 4. The agreement is excellent, and the corre- 


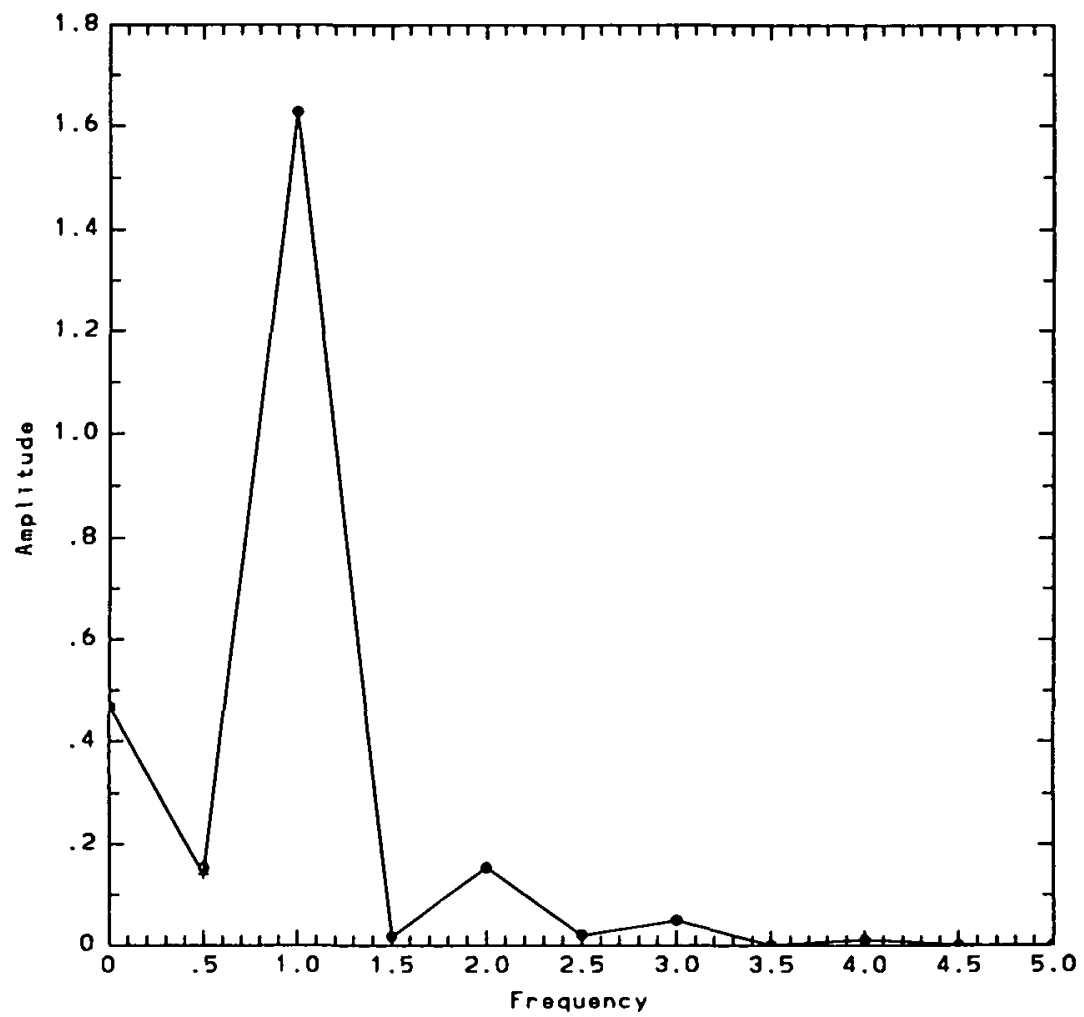

Figure 4. The frequency spectrum of an ensemble average over 16 forcing periods for the nearly-periodic oscillations with $\epsilon=1,2, \omega=0.8700$ and $\delta=0.125$. The joined crosses are the spectral amplitudes greater than 0.01 , and the circles are the spectral amplitudes of the unstable $2 T$-periodic solution at the same parameter values.

sponding agreement between the phases is also excellent, showing that the phase-locked part of the nearly-periodic oscillations is almost identical with the unstable $2 T$-periodic solution at this frequency. The phase-free part of the nearly-periodic oscillations has spectral amplitudes less than 0.01 of the phase-locked oscillations.

When a similar comparison was made at $\omega=0.8705$, closer to the end of the period-doubling sequence than the example in Figure 4, excellent agreement was found between the spectral composition of the averaged $16 T$ interval and the unstable $4 T$-periodic solution at this frequency. The phase locking of the spectral components at frequencies $\omega / 4$ and $3 \omega / 4$ that occurs here is loosened as the forcing frequency is reduced to the value $\omega=0.8700$ of Figure 4. When the frequency is reduced further and a similar comparison made at $\omega=0.868$, the phase locking of the spectral components at frequencies $\omega / 2$ and $3 \omega / 2$ is loosened also. Agreement is found only 
between the spectral composition of the averaged $16 T$-interval and the unstable $T$-periodic solution at this frequency, or in other words, the phase-locking in the nearly-periodic oscillations at this frequency is confined predominantly to the spectral components at integer multiples of $\omega$.

In summary, the nearly-periodic oscillations are characterised by a loosening of the phase-locking that occurs between the spectral components of the exactly periodic oscillations. The loosening increases with an increase in the interval between the forcing frequency and the frequency at the end of the sequence of exactly periodic oscillations. This is consistent with the observation made in the previous investigation [3], that the nearly-periodic oscillations near but beyond the end of a period-doubling sequence retain the dominant features of the exactly periodic oscillations they follow.

Floquet's method (Appendix A) confirms that the strictly-periodic solutions in the neighbourhood in phase space of the nearly-periodic oscillations are linearly unstable, and that the nearly-periodic oscillations themselves are linearly unstable. However, it is clear that the nearly-periodic oscillations are stable in a nonlinear sense, because the nonlinear modification of the unstable linear perturbations results in solutions which makes only small but finite departures in phase space from the corresponding strictly-periodic solutions.

\section{Paired nearly-periodic oscillations}

The oscillations remain nearly-periodic near one or other of the two related, unstable, $T$-periodic solutions as the forcing frequency is decreased from the value $\omega=0.8700$ of Figures 3 and 4 to $\omega=0.8668$, apart from a window of stable, $10 T$-periodic oscillations at $\omega=0.8677$. However, when the forcing frequency is reduced from $\omega=0.8668$ to $\omega=8.8667$, the oscillations become nearly-periodic about both of the related, unstable, $T$-periodic solutions, with intermittent switching between them. The paired nearly-periodic oscillations persist at frequencies down to about $\omega=0.8640$.

The envelope of the paired nearly-periodic oscillations at $\omega=0.8660$ over 200 forcing periods is drawn in Figure 5. The upper curve joins the maximum points of $\theta / \pi$ and the lower curve joins their minimum points. It can be seen that from 10 periods to 30 periods approximately, the oscillations lie between a minimum of about -0.6 and a maximum of about 0.4 , and from 45 periods to 145 periods approximately, they reverse to lie between a minimum of about -0.4 and a maximum of about 0.6 . The latter values are about the same as the minimum and maximum values of $\theta / \pi$ in Figure 2 , and the former values correspond to the related solution $-\theta(t+T / 2) / \pi$. The switching between the two forms of nearly-periodic oscillations in the 


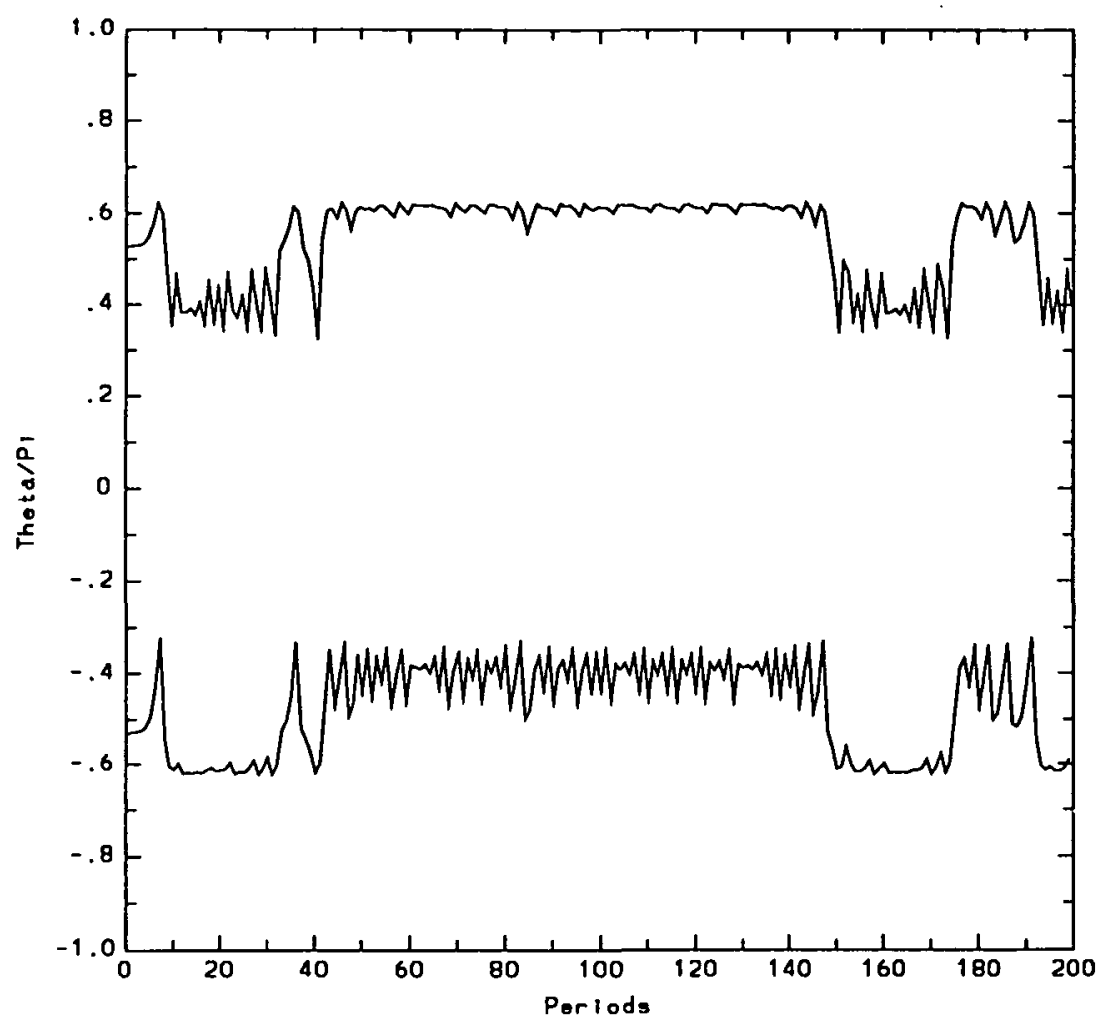

FIGURE 5. The envelope over 200 forcing periods for the paired nearly-periodic oscillations with $\epsilon=1.2, \omega=0.866$ and $\delta=0.125$.

figure is seen to be intermittent, and remains intermittent when the solution is continued to larger times. It is noted that for both forms, the envelope at magnitude 0.6 is rather smoother than the envelope at magnitude 0.4 , but the reason for this difference is not known.

The arguments of [5], when applied to the present phenomenon, suggest that the probable reason for the intermittent switching between the two nearly-periodic states is that the basins of attraction of the two states are interwoven on arbitrarily small length scales, and the basin boundaries are fractal sets. This complicated geometry has extraordinary sensitivity to the noise that is always present in a numerical calculation or in a physical motion.

The paired nearly-periodic oscillations occur in the frequency band $\omega=$ 0.8667 to $\omega=0.8640$ beyond the end of the dominant period-doubling sequence at the forcing amplitude $\epsilon=1.2$. The question arises as to whether they occur beyond the end of other period-doubling sequences at this forcing amplitude, and if so, what form do they take. 
The largest window of stable, periodic oscillations in this frequency neighbourhood is one of $7 T$-periodic, inverted oscillations, beginning with symmetric oscillations from $\omega=0.8407$ to $\omega=0.8420$ and asymmetric oscillations from $\omega=0.8421$ to $\omega=0.8427$. Period-doubling occurs with $14 T$-periodic, inverted oscillations from $\omega=0.8428$ to $\omega=0.8429$ and $28 T$-periodic, inverted oscillations at $\omega=0.8430$. The 7T-periodic, inverted oscillations have the Fourier decomposition (2.1) with $m=7$, where $a_{0}=\pi$ and all even harmonics are zero for the symmetric oscillations, and $a_{0}$ lies near $\pi$ for the asymmetric oscillations. The phase-plane diagrams for each of the two asymmetric oscillations at $\omega=0.8425$ are drawn in Figure 6. Each of the solutions may be obtained from the other by reflecting about both $\theta=0$ (modulus $2 \pi$ ) and $\dot{\theta}=0$, since if $\theta(t)$ is a solution of $(1.1)$, so is $-\theta(t+7 T / 2)$. These two solutions are identical when the oscillations are symmetric. The asymmetric $14 T$-periodic and $28 T$-periodic oscillations have two related forms also, with phase-plane diagrams close to those in Figure 6.

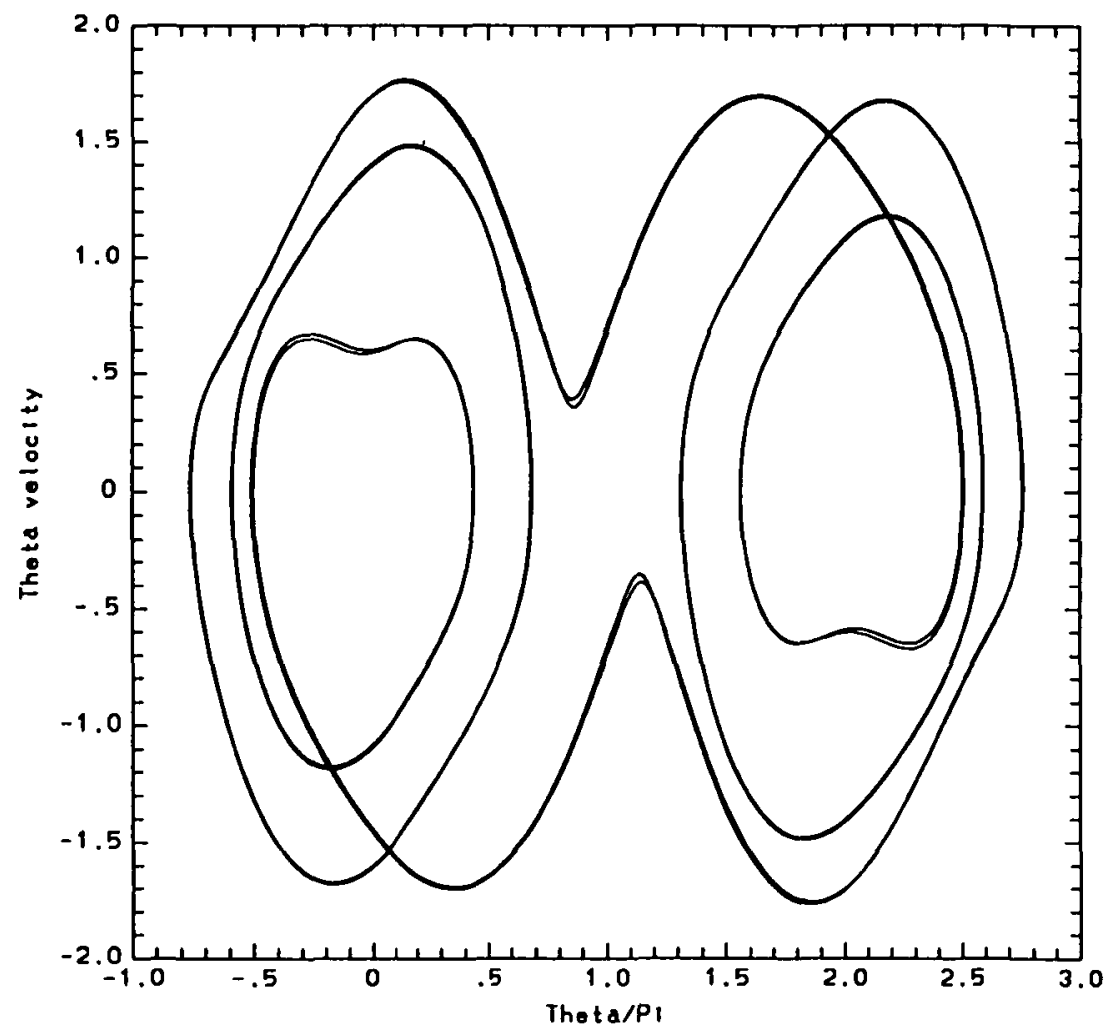

FIGURE 6. The phase-plane diagram, $\dot{\theta}$ agains $\theta / \pi$, for each of the two $7 T$-periodic asymmetric, inverted, stable oscillations with $\epsilon=1.2, \omega=0.8425$ and $\delta=01.25$. 
When the forcing frequency is increased beyond the end of the perioddoubling sequence, the resulting nearly-periodic oscillations remain in the neighbourhood in phase space of one or other of the two related solutions in Figure 6 over the range from $\omega=0.8431$ to $\omega=0.8432$. Paired nearlyperiodic oscillations are found to occur from $\omega=0.8433$ to $\omega=0.8439$, except at $\omega=0.8436$, and the unstable oscillations from $\omega=0.8440$ onwards have no spectral preference to integer multiples of the frequency $\omega / 7$. The intermittency of the paired oscillations at $\omega=0.8436$ was found to be constant with a cycle of 3 oscillation periods, equal to 21 forcing periods. More detailed examination showed that the solution there describes stable, $21 T$-periodic oscillations on a path in phase space between the two unstable, related $7 T$-periodic oscillations.

The two asymmetric solutions in Figure 6 are very close over much of their paths, but two points on the phase-plane diagrams where there is a reasonable separation are the minima and maxima near $\theta=\pi$, with the minima at about $\dot{\theta}=0.4$ and the maxima at about $\dot{\theta}=-0.4$. The locations of these minima and maxima over $\mathbf{3 5 0}$ forcing periods are plotted in Figure 7

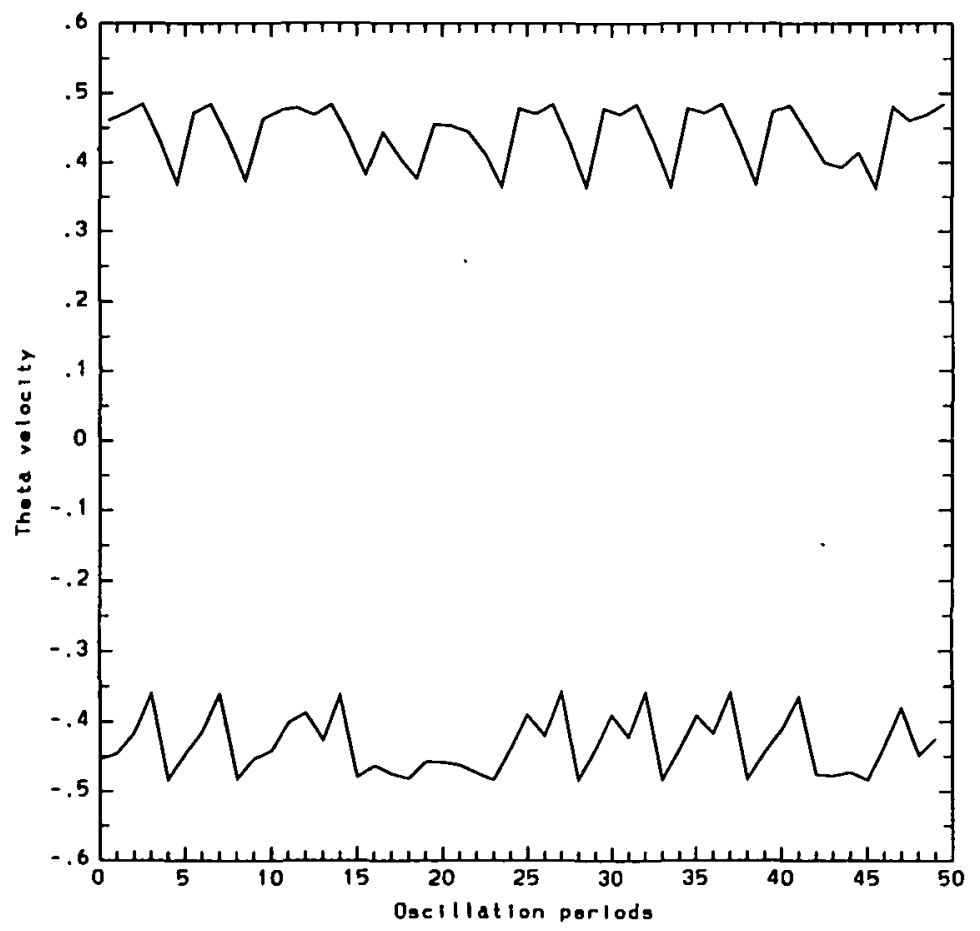

Figure 7. The envelope over 50 oscillation periods, equal to 350 forcing periods, for two stationary values in the $(\theta, \dot{\theta})$ phase plane of the paired nearly-periodic oscillations with $\pi=$ $1.2, \omega=0.8435$ and $\delta=1.25$. 
for the paired nearly-periodic oscillations at $\omega=0.8435$. The 350 forcing periods are equivalent to 50 oscillation periods, and each curve in Figure 7 is formed by joining the 50 stationary values. Although there appears to be faster intermittent switching between the two nearly-periodic oscillations here than in Figure 5, the intermittency is about the same because each oscillation period is equal to 7 forcing periods. Like Figure 5, the curves are smoother where the stationary values are near the larger magnitude than near the smaller magnitude.

\section{Fully chaotic oscillations}

Paired nearly-periodic oscillations are found from $\omega=0.8667$ to $\omega=$ 0.8640 , beyond the dominant period-doubling sequence. When the forcing frequency is decreased further to $\omega=0.8639$, the pendulum not only switches intermittently between the paired nearly-periodic oscillations but also passes intermittently over the top, $\theta=\pi(\bmod 2 \pi)$. It has intervals of low instability in paired nearly-periodic oscillations interspersed with intervals of high instability characterised by repeated passes over the top in both directions. The phase plane diagrams for consecutive intervals of 50 forcing periods at $\omega=0.862$ are drawn in Figures 8 and 9 (see page 166).

Figure 8 begins with 28 forcing periods or paired nearly-periodic oscillations about $\theta=0$, during which the phase difference between the pendulum motion and the forcing of the pivot remains within a narrow range. The range of the phase difference gradually increases after the 28th forcing period until the pendulum passes over the top, $\theta=-\pi$, and oscillates 3 times about $\theta=-2 \pi$. It then returns over the top to oscillate about $\theta=0$ again with phase differences between forcing and response outside the nearly-periodic range. The oscillations are sufficiently unstable that they repeat the traverse each way over the top, then continue to go over the top in both directions during the next 50 forcing periods, illustrated in Figure 9. When the solution is continued at this frequency, it is found to have intervals like that in Figure 8 in which the motion consists of paired nearly-periodic oscillations within a narrow range of phase differences, interspersed with intervals like Figure 9 of more unstable oscillations passing intermittently over the top with the whole range of phase differences between the forcing and the response.

The appearance of the $(\theta, \dot{\theta})$ phase plane diagram changes as the motion changes from a single closed curve for periodic oscillations (Figures 2 and 6), to a narrow band of curves for nearly-periodic and paired nearly-periodic 


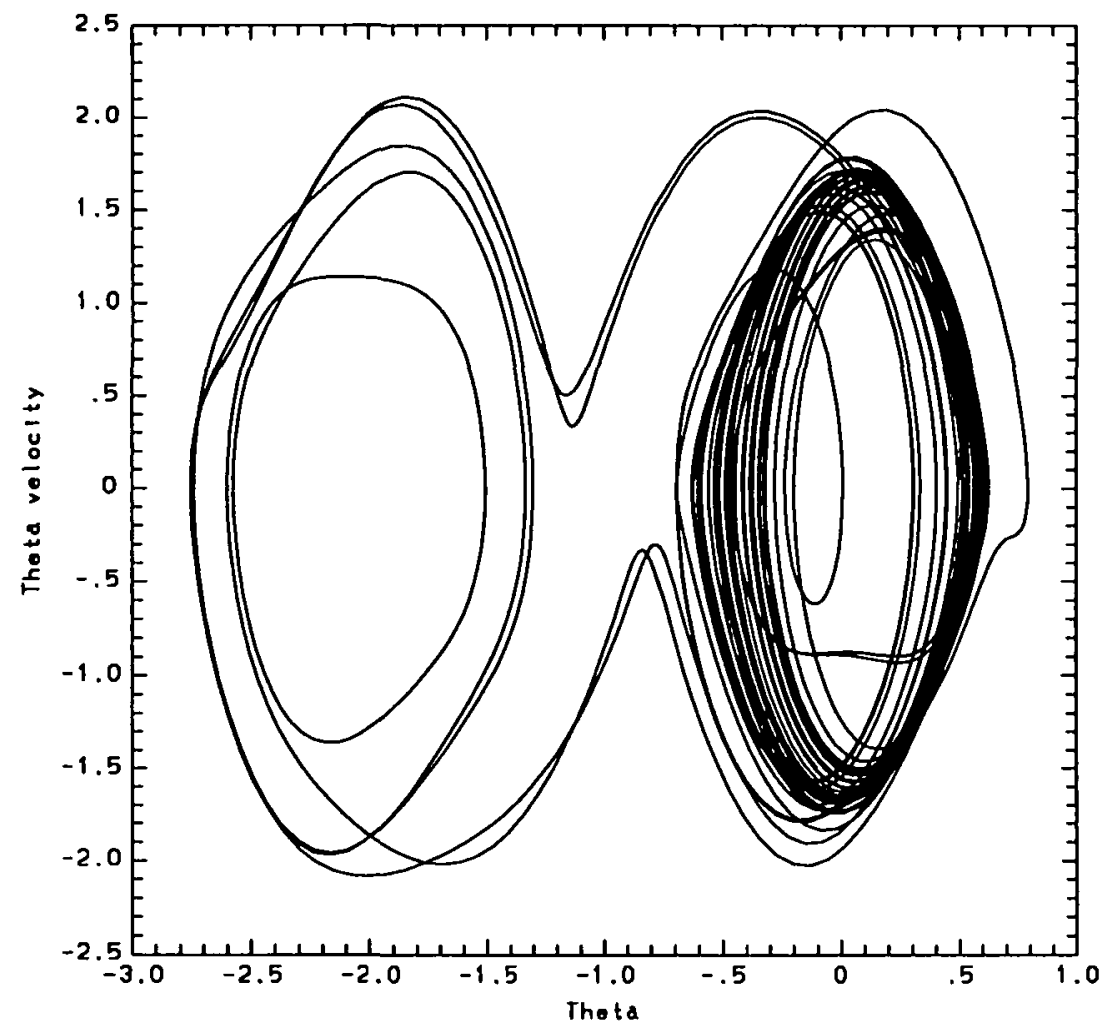

FIGURE 8. The phase-plane diagram, $\dot{\theta}$ against $\theta / \pi$, over 50 forcing periods with $\epsilon=1.2$, $\omega=0.862$, and $\delta=0.125$.

oscillations (Figure 3), to curves spread over much of the given part of the phase plane (Figures 8 and 9) for fully chaotic oscillations (particularly when $\theta$ is reduced to modulus $2 \pi$ ). A good indicator for the type of pendulum motion is the proportion of the given part of the phase plane through which the phase plane curve passes. This is easily found numerically by placing a fine grid on the phase plane and counting the number of squares in the grid through which the curve passes in a large time, with multiple passes through the same square being counted once only. The angle $\theta$ is reduced to modulus $2 \pi$ so that complete circuits of the pivot contribute larger magnitudes of $\dot{\theta}$ without additional values of $\theta$. The area of the $(\theta, \dot{\theta})$ phase plane through which the curve passes, arbitrarily normalised, is shown in Figure 10 (see page 167) for the forcing frequency range $\omega=0.80$ to $\omega=0.88$.

This count of the number of squares in the phase plane is a measure of the entropy of the oscillations ([1], pp. 87-88), a concept with the same sense as in statistical mechanics. A figure showing the entropy variation for the 


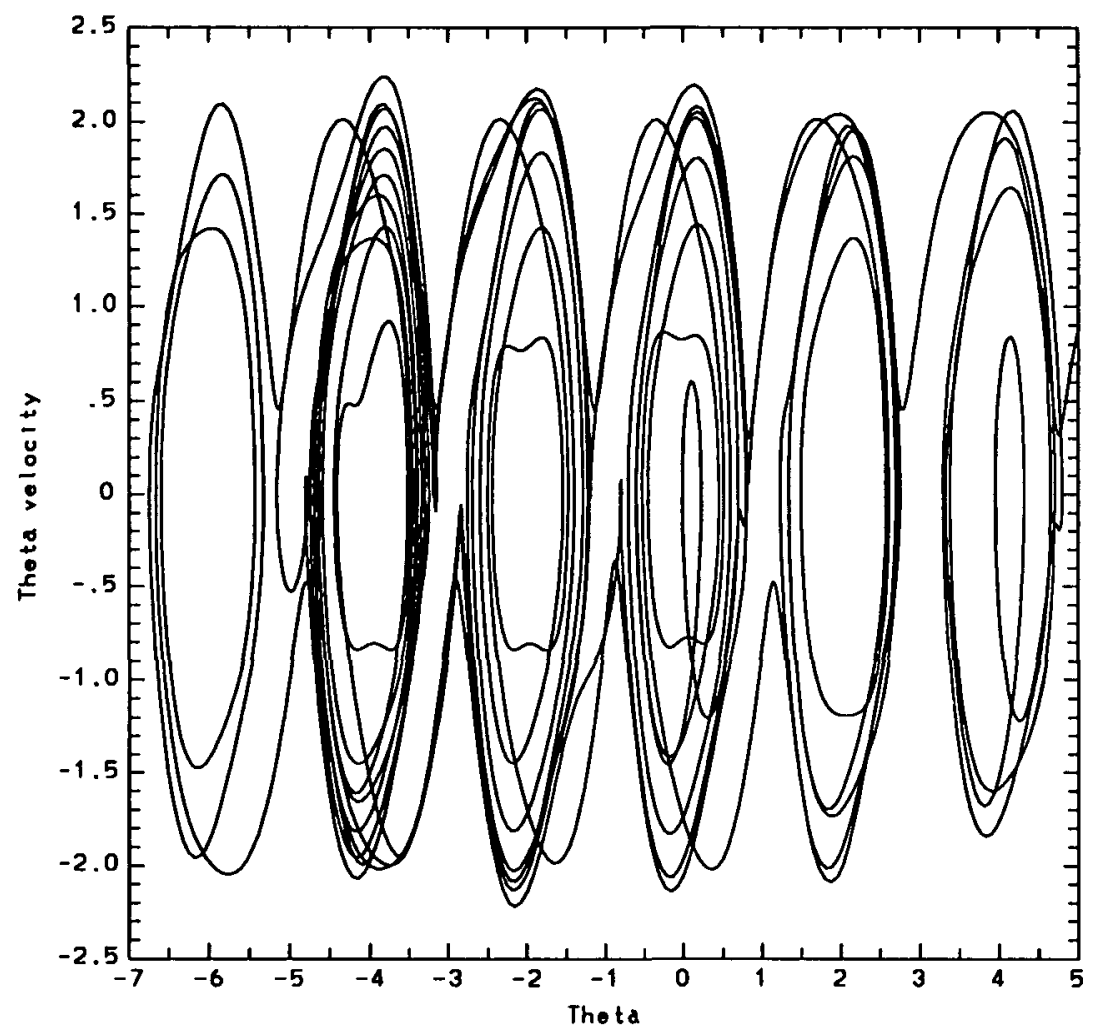

FIgURE 9. The phase-plane diagram, $\dot{\theta}$ against $\theta / \pi$, over the next 50 forcing periods from Fig. 8, with $\epsilon=1.2, \omega=0.862$, and $\delta=01.25$.

one-parameter logistic map ([1], Figure 4.10) bears a striking similarity to Figure 10 even though the difference equation for the logistic map is apparently unrelated to the differential equation (1.1) above.

The stable oscillations are $2 T$-periodic from the right of the figure to the frequency $\omega=0.8732$, at which period-doubling occurs and the area traversed in the phase plane almost doubles, from 0.004 to 0.0075 . Perioddoubling occurs again at $\omega=0.8712$, at which the area traversed in the phase plane almost doubles again to 0.013 . These areas are all very small because the motion is represented by a single closed curve in the $(\theta, \dot{\theta})$ phase plane. Similarly, all other windows of exactly-periodic oscillations in the frequency range of Figure 10 are described by small phase plane areas, appearing as downward spikes in the figure. The oscillations in these windows are tabulated in Appendix B.

Apart from the downward spikes described above, the area remains less than 0.08 but has an upward trend from $\omega=0.8707$ to $\omega=0.8668$. This is 


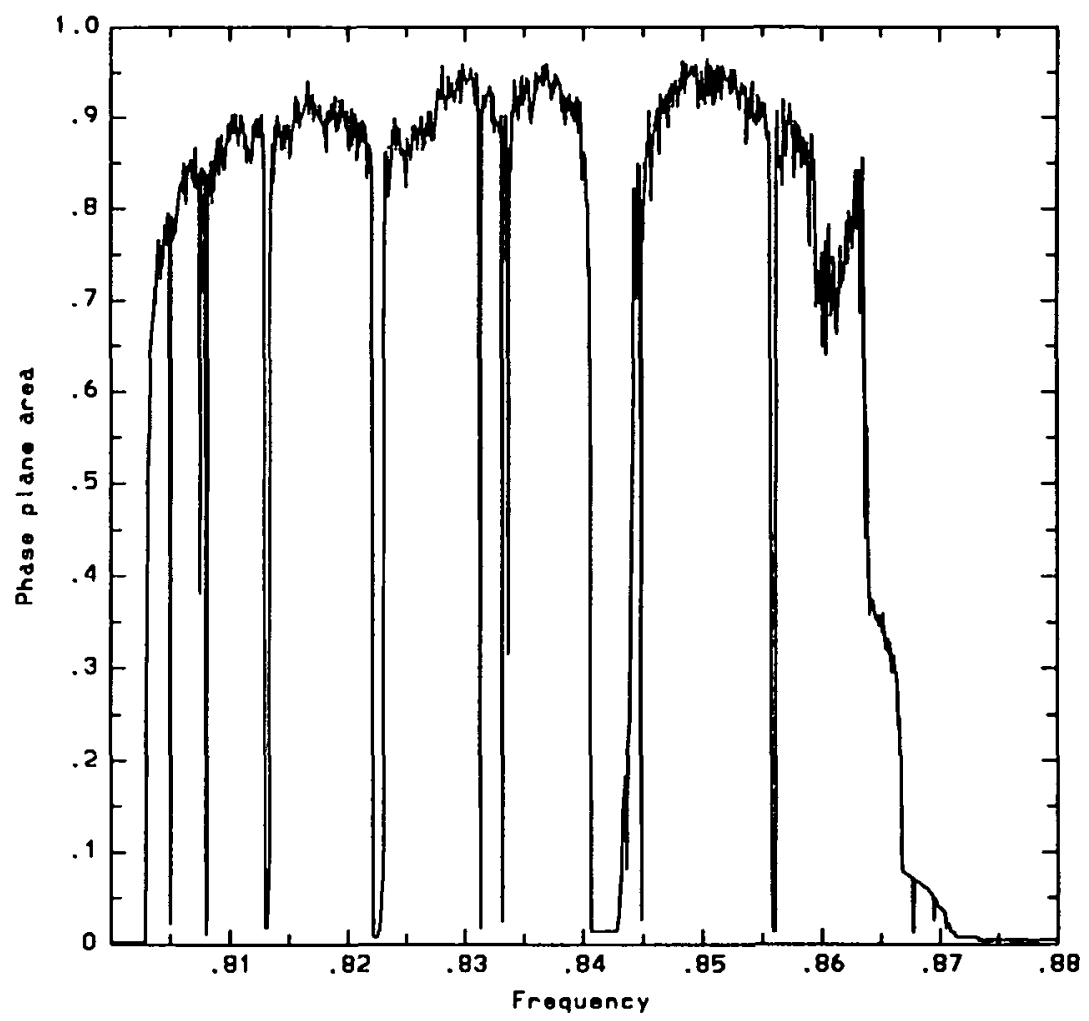

FIGURE 10. The proportion of the $(\theta, \dot{\theta})$ plane arbitrarily normalised, through which the phase-plane diagram passes, with $\epsilon=1.2$ and $\delta=0.125$.

the range of nearly-periodic oscillations described in Section 3 and illustrated in Figure 3, in which the motion remains near one, but not both, of the two related unstable exactly-periodic oscillations. The area increases discontinuously at $\omega=0.8668$, then has an upward trend from 0.29 to 0.37 between $\omega=0.8667$ and $\omega=0.8640$. This is the range of paired nearly-periodic oscillations described in Section 4, in which the motion switches intermittently between both of the two related unstable exactly-periodic oscillations.

Over much of the remainder of the frequency range of Figure 10, the area lies in the range 0.7 to 0.95 , apart from the downward spikes indicating periodic oscillations. The values for the area exhibit some scatter because of the intermittency between intervals of low instability and intervals of high instability in the fully chaotic oscillations, as illustrated in Figures 8 and 9. Although the counting of squares in the phase plane was made over 1000 forcing periods, counts over consecutive intervals of 1000 forcing periods at the same frequency yielded different values for the count within the scatter 
illustrated. The scatter could have been reduced by using a larger counting interval, with correspondingly more computation. Lower values of the area occur in the frequency range $\omega=0.862$ to $\omega=0.8595$, including the frequency $\omega=0.862$ of Figures 8 and 9 . This appears to be associated with longer intervals of nearly-periodic oscillations of low instability than occur elsewhere.

The large window of $7 T$-periodic oscillations, described in Section 4 and illustrated in Figure 6, can be seen near the centre of Figure 10. It is noted that the area on the right of this window in Figure 10 increases steadily from $\omega=0.8429$ to $\omega=0.8439$, apart from the downward spike of $21 T$. periodic oscillations at $\omega=0.8436$. This range spans, in order, the perioddoubling sequence, the nearly-periodic oscillations, and the paired nearlyperiodic oscillations described by Figure 7. Similar ranges of increasing area can be seen on one side of other windows in Figure 10, such as the window of $4 T$-periodic running oscillations near $\omega=0.8225$. These also describe period-doubling followed by the gradual breakdown of the phase locking of the exactly-periodic oscillations.

\section{Nearly-periodic oscillations with intermittent switching}

There is a transition on the left of Figure 10 from chaotic oscillations to stable $T$-periodic symmetric oscillations at $\omega=0.8029$. This is the turning point $\omega=\omega_{+}$for $T$-periodic symmetric oscillations, in the notation of [3], illustrated in [3], Figure 1c. Stable $T$-periodic symmetric oscillations exist at all frequencies less than $\omega_{+}$.

It can be seen in Figure 10 that the area traversed by the phase plane diagram increases from 0.35 at $\omega=0.8030$ to a fully chaotic level of about 0.8 at $\omega=0.8050$. The oscillations in this frequency range lie in a transition between the stable $T$-periodic symmetric oscillations and the fully chaotic oscillations. The phase plane diagram over 100 forcing periods at $\omega=0.8030$ is drawn in Figure 11. The motion consists of nearly-periodic oscillations about $\theta=0$ switching over a short disturbed time interval to nearly-periodic oscillations about $\theta=2 \pi$. The motion over the next 100 forcing periods consists almost entirely of nearly-periodic oscillations about $\theta=2 \pi$, with only a short disturbed time interval in the middle when the pendulum makes two oscillations about $\theta=0$ before returning. This motion is described as nearly-periodic oscillations with intermittent switching. As the frequency is increased from the value $\omega=0.8030$ of Figure 11 , the rate of intermittent switching and the range of values of $\theta$ both increase until fully chaotic oscillations are attained. 


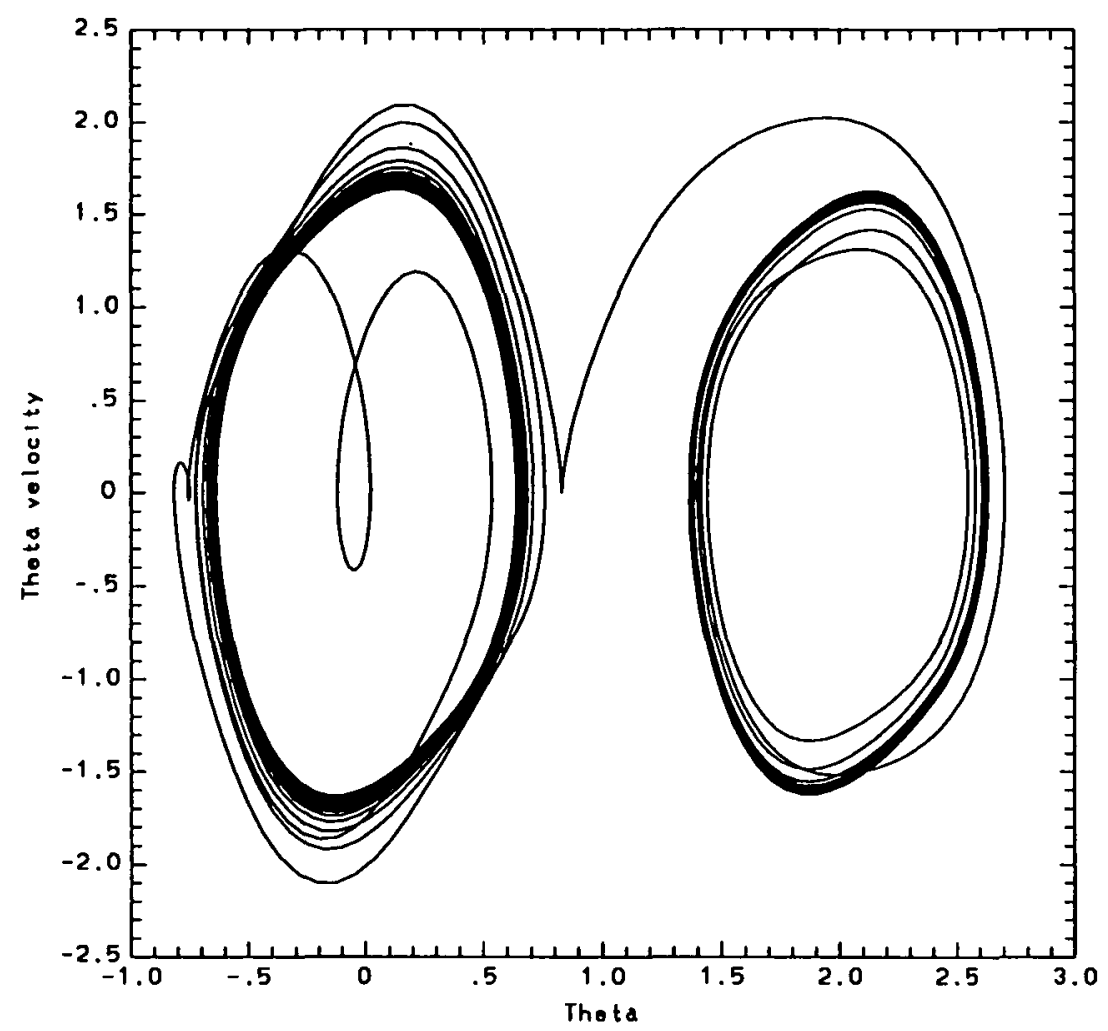

Figure 11. The phase-plane diagram, $\dot{\theta}$ against $\theta / \pi$, over 100 forcing periods with $\epsilon=$ 1.2, $\omega=0.8030$, and $\delta=0.125$.

The major difference between these oscillations and the nearly-periodic oscillations of Section 3 or the paired nearly-periodic oscillations of Section 4 is that unstable oscillations exist at the frequencies of Section 3 and Section 4 , but not at the present frequencies. The oscillations in Section 3 and Section 4 remain in the neighbourhood in phase space of one or both of the two related, weakly-unstable periodic solutions at the same frequency, without the pendulum passing over the pivot. The oscillations here remain for extended time intervals in the neighbourhood in phase space of the periodic solution at the turning-point frequency, different from the forcing frequency, but the pendulum passes intermittently over the pivot at other times.

The common feature between these oscillations and those in Section 3 and Section 4 is that the motion is only weakly chaotic, in the sense that it continues to display a strong ordered element. It is the ordered element that results in the phase plane diagram passing through a smaller proportion of the phase plane area than fully chaotic oscillations, as is indicated by Figure 
10. Intermittent attractors, such as that illustrated in Figures 8,9 and 11 are investigated in some detail in [5], where the intrinsic intermittency is illustrated in terms of transitions among basins of attraction for solutions of (1.3) with positive and negative mean angular velocities. The present investigation suggests that intermittency is a more general phenomenon in the chaotic breakdown of periodic oscillations of forced, damped pendulums.

\section{Discussion}

The primary purpose of this investigation is to examine the nature of the chaotic motion in the frequency neighbourhood of stable periodic motion for the very simple dynamical system consisting of a forced, damped, coplanar pendulum. It was found that the chaotic motion retains major elements of the periodic motion at neighbouring frequencies, and does not become fully chaotic unless the forcing frequency differs sufficiently from those in the periodic range. The weakly chaotic motion is found beyond the end of a period-doubling sequence and also beyond a turning-point stability boundary. It persists for a larger frequency range in the first case than it does in the second case, presumably because the loss of stability is more abrupt in the second case.

Similar properties are known for much more complex, forced dynamical systems. The review by Berger and Wille [2] of periodic flow phenomena in the laboratory and in the atmosphere describes a number of properties parallel to those investigated here. Strictly-periodic vortex shedding occurs for two-dimensional flow around a circular cylinder at low Reynolds numbers $\left(\sim 10^{2}\right)$ and low turbulence levels, yet a periodic vortex-street wake still persists in a fully turbulent flow around bluff bodies at Reynolds numbers greater then $10^{6}$. The bluff body forces the wake periodically, resulting in recognisable near-periodicity in the fully turbulent wake. The review reports 'controversial' experiments by Tritton [6] in which the wake behind a circular cylinder was observed to jerk between two vortex street modes for a particular range of Reynolds number and turbulence level. This behaviour is similar to that of the paired nearly-periodic oscillations of Section 4, and may not have been due to some non-uniformity in the flow, as was suggested at the time.

\section{Appendix A: Floquet's method}

Floquet's method is used to calculate the linear stability of solutions to (1.1) and to determine the initial form of their instability when it occurs. 
The method is summarised here because its predictions of instability must be interpreted carefully to correspond to actual instability, as determined by step-by-step integration of (1.1).

If $\theta=\theta_{0}(t)$ is a solution of $(1.1)$, perturbations $\hat{\theta}(t)$ to the solution satisfy the pair of first order differential equations

$$
\begin{gathered}
\dot{\hat{\theta}}=p, \\
\dot{p}=-2 \delta p-\hat{\theta}\left(\cos \theta_{0}+\epsilon \sin \theta_{0} \sin \omega t\right),
\end{gathered}
$$

after linearisation in $\hat{\theta}$. The linear stability of an $m T$-periodic solution (2.1) is calculated by expansion of $p$ and $\hat{\theta}$ in Fourier series of the same form as (2.1) except that the coefficients are time-dependent, followed by substitution of the series for $\theta_{0}, p$ and $\hat{\theta}$ into (A1) and (A2). A Fourier analysis (using Fast Fourier Transforms) is made of the resulting equations, with the assumption that the Fourier coefficients depend only slowly in time, to obtain a set of $4 N+2$ linear first order differential equations with constant coefficients for the $4 N+2$ independent Fourier coefficients in the expansions of $p$ and $\hat{\theta}$. The set is solved numerically by setting the time dependence of the $4 N+2$ Fourier coefficients proportional to $\epsilon^{\lambda \omega t / m}$ followed by calculation of the eigenvalues $\lambda$ and eigenvectors of the resulting square matrix of order $4 N+2$ (using the NAG subroutine F02AGF).

The eigenvalues have the form

$$
\lambda=\lambda_{r}+i \lambda_{i},
$$

which substituted into equations of the same form as (2.1) yield solutions

$$
\hat{\theta}=\sum_{k=0}^{N}\left[\alpha_{k} e^{\left(\lambda_{r}+i \lambda_{i}\right) \omega t / m} \cos (k \omega t / m)+\beta_{k} e^{\left(\lambda_{r}+i \lambda_{i}\right) \omega t / m} \sin (k \omega t / m)\right]
$$

where all $\alpha_{k}, \beta_{k}$ are constants. Although this is a formal solution of the set of $4 N+2$ linear first order differential equations, it is inconsistent with the assumption of slow time dependence in the Fourier analysis yielding these equations unless $\lambda_{i} \leq 1 / 2$.

Provided $N$ is taken sufficiently large, stability calculations for particular examples produce either integer values for $\lambda_{i}$, or integer values plus $1 / 2$ when period-doubling occurs. Instability occurs, in principle, when $\lambda_{r}$ is positive. However, step-by-step integration of (1.1) from initial conditions in the neighbourhood of a given solution shows that instability of the solution occurs in practice only when there are eigenvalues for which $\lambda_{r}>0$ and $\lambda_{i} \leq 1 / 2$. Eigenvalues for which $\lambda_{r}>0$ but $\lambda_{i}>1 / 2$ have no physical 
application for the reasons above, and the apparent instability proves to be spurious.

\section{Appendix B: Windows of periodic oscillations}

The asymptotic solutions of (1.1) were calculated for the frequency range $0.80 \leq \omega \leq 0.88$, at frequency intervals of 0.0001 , to obtain the data for Figure 10. Zero initial conditions, $\theta=\dot{\theta}=0$, were used for each calculation, the solution for the first $\mathbf{5 0 0}$ forcing periods was discarded, and the data for Figure 10 was obtained from the next 1000 forcing periods. The downward spikes in the figure indicate windows of periodic oscillations, which are tabulated below. Not all windows of stable, periodic oscillations in the frequency range were necessarily found, either because the window width is too small for the frequency interval used, or because the numerical solution did not enter the basin of attraction in phase space of the period solution.

Frequency

0.8050

0.8075

$0.8080-0.8081$

$0.8130-0.8133$

$0.8221-0.8230$

0.8313

0.8332

0.8337

$0.8407-0.8439$

0.8436

0.8448

$0.8559-0.8561$

0.86677

0.8695

0.8706
Periodic oscillations

Period $11 T$

Period $21 T$

Period $5 T$, mean angular velocity $\omega / 5$

Period $9 T$, inverted

Period $4 T$, mean angular velocity $\omega / 4$

Period $9 T$, inverted

Period 16T, inverted

Period 16T, inverted

Period $7 T$, inverted

Period $21 T$, inverted

Period $11 T$, mean angular velocity $\omega / 11$

Period $7 T$, inverted

Period $10 T$

Period $16 T$

Period 20T

\section{References}

[1] Gregory L. Baker and Jerry P. Gollub, Chaotic Dynamics (Cambridge University Press, Cambridge, 1990).

[2] Eberhard Berger and Rudolf Wille, "Periodic flow phenomena", Ann. Rev. Fluid Mech. 4 (1972) 313-340. 
[3] Peter J. Bryant and John W. Miles, "On a periodically-forced, weakly damped pendulum: Part 2: Horizontal forcing", J. Austral. Math. Soc. Ser. B. 32 (1990) 23-41.

[4] Mitchell J. Feigenbaum, "Quantitative universality for a class of nonlinear transformations", J. Stat. Phys. 19 (1978), 25-52.

[5] E. G. Gwinn and R. M. Westervelt, "Fractal basin boundaries and intermittency in the driven pendulum", Phys. Rev. A. 33 (1986) 4143-55.

[6] D. J. Tritton, "A note on vortex streets behind circular cylinders at low Reynolds number", J. Fluid Mech. 45 (1971) 203-8. 